\title{
Student vor 50 Jahren
}

\section{von Horst Tietz}

Die folgende Rede hat Horst Tietz am 12.4.1996 auf einem Kolloquium anläßlich seines 75. Geburtstags gehalten. Er stellte seine Rede unter das Motto: Das Lebensgefühl des Menschen ist nicht etwa seinem Lebensstandard proportional - sondern dessen Differentialquotienten!

Das ärmste Semester meines Lebens, das erste Nachkriegssemester, war das schönste: es begann am 6. November 1945 an der Universität Hamburg.

Erich Hecke - obgleich sterbenskrank - las „Lineare Differentialgleichungen“, d.h. Fuchssche Theorie und Anwendung besonders auf Kugelfunktionen und Darstellungen der Orthogonalen Gruppen. Die Atmosphäre ist heute unfaßbar, wie hungrig die ausgemergelten, zerlumpten Gestalten in knisternder Spannung der faszinierenden Vorlesung dieser großartigen Persönlichkeit folgten: Hecke verband Wärme und Würde und gab damit einem Menschenbild wieder Leben, das seit der Nazizeit verschwunden schien.

Unvergessen eine Unterbrechung: es war wohl im Januar 1946, daß Hecke, der beim Sprechen gerne über die Pappverkleidung der zerschossenen Mauern und Fenster des Hörsaals hinweg auf die Straße sah, mit freudiger Überraschung mitten im Satz abbrach, die Kreide hinlegte und mit den Worten „ich habe lieben Besuch bekommen ", auf die Straße eilte und dort Ehrhard Schmidt umarmte, der auf seiner Flucht aus Berlin von seinem Schüler Thomas von Randow, dem heutigen Zweistein im Zeit-Magazin, nach Hamburg gebracht worden war. -

Hecke stellte ferner in einer Spezialvorlesung seine Untersuchungen über Modulformen vor; in seiner ausgehängten Vorlesungsankündigung stand „Nur für Erwachsene“, und ich war sehr stolz, daß Hecke mich dazu aufforderte; und so saß ich zwischen Assistenten und Dozenten in Heckes eigener Werkstatt.

Auch an weitere Veranstaltungen, die ich besuchte, erinnere ich mich gerne: Zassenhaus: „Raumgruppen“, Weissinger „Integralgleichungen“, Noack ließ Holmogoroffs "Wahrscheinlichkeitsrechnung" in seinem Seminar referieren, und Maak trug für Lehramtskandidaten das Buch „Zahlen und Figuren“ von Rademacher-Töplitz vor. Mit höflichem Zweifel hörte er zu, als ich ihm zeigte, wie ein zahlentheoretischer Beweis erheblich vereinfacht werden konnte.

Maak war ein Mensch mit skurrilem Humor: von ihm habe ich die schönsten Formulierungen: für den Mathematiker ist es das Schlimmste, wenn er nicht weiß, worüber er nachdenken soll,-- und ich wolle ich wäre zwei junge Hunde und könnte miteinander spielen!

Ihm passierten Pannen von erlesener Delikatesse; die schönste ist wohl die mit seinem Briefkastenschlüssel: er hatte ihn bei Harald Bohr in Kopenha- gen vergessen und mochte bei seiner Rückkehr den vollen Briefkasten nicht aufbrechen; seiner schriftlichen Bitte um Zusendung des Schlüssels kam Bohr umgehend nach; er schickte ihn - in einem Brief - in den Briefkasten.

Mit Zassenhaus und Maak gemeinsam fuhr ich abends oft mit der S-Bahn nach Othmarschen, einem Elbvorort von Hamburg, wo viele Ausgebombte Unterkunft gefunden hatten. Als eine der häufigen Stromsperren unsere Fahrt unterbrach und uns zu unabsehbarem Warten zwang, vertrieben wir uns in dem dunklen, kalten, überfüllten Abteil stehend die Zeit mit immer raffinierteren Denksportaufgaben; Herr Maak setzte diesem Wettstreit die Krone auf: „ein Jäger, ein Begleiter und ein Hund fliehen vor einem Löwen in ein Haus, in dem nur ein Schrank mit vier Füßen steht; sie klettern auf den Schrank, der Löwe kommt herein und legt sich hin; als er eingeschlafen war, sagt der Begleiter plötzlich: hier sind sieben Füße im Haus! - Wieso???" Nach langem, scharfsinnigem, vergeblichem Raten bat der gemarterte Zassenhaus schließlich flehentlich um Erlösung. Vielleicht hat nur die Finsternis Handgreiflichkeiten verhindert, als Maak ihn mit dem ihm eigenen erstaunten vorwurfsvollen Ton wie einen Kandidaten, der eine triviale Frage nicht beherrscht, belehrte: der Begleiter konnte nicht zählen ...

Zassenhaus war ein heiterer Mensch anderer Art und lachte sehr gern; aber Humor ohne Problem war ihm schwer erreichbar. Ich erzählte ihm einen der ersten Nachkriegswitze: in Düsseldorf fragte eine alte Dame in der Straßenbahn nach dem Adolf Hitler-Platz; als der Schaffner sie belehrte, der heiße jetzt Graf Adolf-Platz, meinte sie aus vollem Herzen: „Ach, der Gute, das hat er auch verdient!" noch während er vor Lachen nach Luft rang, fragte Zassenhaus: „und was hat da der Schaffner gesagt?"

Zassenhaus, der für die weltfremde Mathematik in Deutschland keine Zukunft sah, hatte eine Denkschrift für die Errichtung eines „Institutes für Praktische Mathematik" erstellt. Da er aber wenig Hoffnung für den Erfolg seines Planes hatte, streckte er schon seine Fühler in die angelsächsische Welt aus.

Da konnte ich mich nützlich machen, und das kam so: demokratische Gremien entstanden damals durch Urzeugung, und in der Erwartung, daß ein studentisches Gremium bei der britischen Militärregierung leichter Gehör finden würde als die NS-verdächtige 
Professorenschaft, hatte sich - schon vor meiner Ankunft im August - als Vorgänger des Asta ein Zentralausschuß Hamburger Studenten, kurz ZA, gegründet, an dessen Beratungen ich bald teilnahm. Ich interessierte den ZA für das Projekt von Zassenhaus, und nach einem Vortrag von ihm wurde ich beauftragt, beim englischen Hochschuloffizier dafür zu werben.

Ich bilde mir ein, zum Erfolg des Projektes beigetragen zu haben: das Institut wurde errichtet, aber doch zu spät, als daß Zassenhaus's Auswanderung hätte gestoppt werden können.

Im ZA übernahm ich das Sozialreferat. Zwei meiner Aktivitäten habe ich in Erinnerung:

zum einen erreichte ich, daß Studenten auf Behörden und Ämtern nicht mehr als arbeitslos, sondern als erwerbslos geführt wurden;

zum anderen wollte ich etwas gegen die unüberwindlichen Schwierigkeiten tun, denen Studierwillige dadurch ausgesetzt waren, daß für die Zulassung zum Studium die Wohnberechtigung in Hamburg notwendig war. Diese konnte man zwar erwerben, wenn man ein Jahr lang bei der Trümmerbeseitigung mitgearbeitet hatte, was aber ohne Unterkunft eben unmöglich war; das brachte mich auf den Gedanken, daß man ja die massiv-gemauerte, unbeschädigte Tribüne 1. Klasse auf der Horner Pferderennbahn (die anderen Tribünen waren offene Gebäude) mit Feldbetten und Kanonenöfen als Unterkunft für etwa 150 potentielle Studenten herrichten könne. Erstaunlicher Weise fand ich bei den Ämtern und Behörden offene Ohren und aktive Hilfe für diesen Plan, und nach kurzer Zeit bot der ZA den künftigen Kommilitonen stolz dies gemachte Nest an - gemeldet hat sich kein einziger! - Deutschland war eben ein Volk von Vagabunden, und die Spanne von einem Jahr war wohl zu lang, um sie in die Planung einer $\mathrm{Zu}$ kunft einbeziehen zu können, die als schwarze, undurchsichtige Wand vor jedem stand. Vielleicht hat mich diese herbe Enttäuschung vor dem Entschluß bewahrt, "Politiker zu werden ..."

Es war ein ziemliches Wagnis gewesen, ein Vierteljahr nach Kriegsende aus der relativen Geborgenheit des kaum zerstörten Marburg, von wo ich gekommen war, in die Trümmerstadt Hamburg zu ziehen: es ist aber meine Heimatstadt, und man munkelte, daß die Hansische Universität vor der Marburger geöffnet werden sollte; vor allem hatte es mich aber zu Hecke und Zassenhaus gezogen, die mich in der Nazi-Zeit so sehr gestützt hatten: sie hatten mich aufgefordert, bei ihnen schwarz zu hören, als ich im Dezember relegiert wurde; Hecke, wohl der einzige Professor, der niemals die Hand zu dem befohlenen „Deutschen Gruß“ erhoben hatte, beendete mitten im Sommersemester 1942 seine „Zahlentheorie“ und zahlte seinen Hörern das Kolleggeld zurück, als mir der Besuch der Universität zu gefährlich wurde, und Zassenhaus arbeitete danach noch privat mit mir - aber schließlich hatte ich ja noch einen Band der Mathematischen Annalen zurückzugeben, den ich 1943 vor der Ausbombung aus dem Seminar entliehen und auf dem Weg durch das Inferno wie meinen Augapfel gehütet hatte. - Das Leben in Hamburg aber ohne Familie und ohne Heim war hart. Obgleich ich reiche menschliche Zuwendung und Hilfe erfuhr, ging ich nach dem beglückenden ersten Nachkriegssemester nach Marburg zurück, zumal Heckes zunehmender körperlicher Verfall meine Arbeit bei ihm ausschloß, und Zassenhaus's Emigrationsplan immer konkreter wurde. - Und in Marburg konnte ich endlich gemeinsam mit meinem Freund Grau, unserem Philosophen, studieren!

Am Tag nach meinem 25sten Geburtstag, an dem mich übrigens eine junge Dame mit einem Blumenstrauß überraschte, fuhr ich also nach Marburg zurück. Fahren darf man nicht in heutigem Sinn verstehen: An Eisenbahn und Post dachte noch niemand; das bißchen, was lief, wurde von besonders lizensierten „Fuhrunternehmern" geleistet, die auf ihren Fahrten - legal oder illegal - Passagiere und Briefe mitnahmen.

Und das war abenteuerlich! Meine nächtliche Fahrt über Land in einem ausgeschlachteten Wehrmachtsbus, der Material für die Marburger Kliniken transportierte, werde ich nie vergessen; ich weiß nicht, wo wir waren, als ein ersticktes Stöhnen uns vier Passagiere, die auf und zwischen Säcken und Kisten zu schlafen versuchten, hochfahren, die Türen aufreißen und aus dem noch bremsenden Wagen herausfallen ließ: erst als es dämmerte, stellten wir fest, daß ein Papiersack, der Kunstpfeffer enthielt, geplatzt war.

Das kaum zerstörte Marburg übte starke Anziehung aus auf die Ströme der Heimkehrer, Flüchtlinge und Heimatlosen. Entsprechend bunt zusammengewürfelt war die Studentenschaft. Die politische Differenzierung spielte eine größere Rolle als in Hamburg, wo die Probleme des Überlebens im Vordergrund standen. Frühere Wehrmachtsoffiziere erkannte man an einer deutlichen Zackigkeit; von ihnen sagten die Physiker: wenn von „Größen höherer Ordnung" die Rede ist, knallen die mit den Hacken! Als ich erfuhr, daß ein Kommilitone im Suff verraten hatte, er sei SS-Offizier gewesen, sprach ich ihn an und erklärte ihm, daß und warum ich keinen Kontakt mehr mit ihm zu haben wünsche; sehr viel später erfuhr ich, daß er, inzwischen OSD, mich als seinen Freund bezeichnete.

Ziemlich heftig war der politische Kampf unter den Professoren: der Rektor, der Philosoph Ebbinghaus, der die Universität entnazifizieren wollte, hatte sich sogar mit dem Germanisten Mitzka auf offener Straße Ohrfeigen geboten. - Trotz seiner Rektorwürde tat er übrigens etwas, was viele taten: er hob die Kippen amerikanischer Zigaretten von der 
Straße auf, was in der Öffentlichkeit hämische Beachtung fand; der Theologe Ernst Benz faßte dies in den schönen Schüttelvers: des Rektors Auge fester schielt, erblickt er eine Chesterfiel!

Mathematisch war an der Philipps-Universität nicht viel los: ein Ordinariat, die Dozentur und die Assistentenstelle waren vakant. Die Dozentur wurde erst 1948 durch Hans-Heinrich Ostmann und 1950 durch unseren Wolfgang Rothstein besetzt. Alleiniger Ordinarius war Kurt Reidemeister, Exordinarius für angewandte Mathematik mein späterer Doktorvater Maximilian Krafft, und mit der Hilfsassistentenstelle, die mit 198,- RM dotiert war, hatte man Herbert Grötzsch abgespeist, der in Marburg Fuß zu fassen versuchte, als die benachbarte Uni Gießen, aus der ihn 10 Jahre vorher, gleich nach seiner Habilitation, die Nazis hinausgeworfen hatten, geschlossen vorfand.

Diese drei Dozenten waren Originale, jeder auf seine Art.

Der schöngeistige und weltfremde Ästhet Reidemeister, der 1933 seine Verachtung gegenüber den plebejischen Massen der Nazis in einer Vorlesung offen erklärt hatte und prompt von Königsberg nach Marburg strafversetzt worden war, kümmerte sich im Fahrwasser seines Freundes Ebbinghaus mehr um Politik als um Mathematik und fuhr bald auf Einladung von Siegel, mit dem er damals noch befreundet war, für ein Jahr nach Amerika. Seine Frau, eine bekannte Porträt-Photographin (manche Bilder aus der Hilbert-Biographie von Constance Reid stammen von ihr) begleitete ihn. Als Mucki und Pinze, wie nicht nur sie sich nannten, wiederkamen, entspann sich zwischen ihm und Ebbinghaus eine Dauerkontroverse über das Raumproblem, und zu beliebigen Tag- und Nachtzeiten wurde ich zu Ebbinghaus zitiert, um ihm den Euklid zu erklären.

Krafft war das Vorbild des Vaters in den bekannten Karikaturen "Vater und Sohn“ von E.O. Plauen dieser hatte eine Nachbarstochter von Krafft geheiratet und in Ruhe seinen Nachbarn beobachten können. - Krafft kehrte in der Vorlesung seinen Hörern den Rücken zu, begann mit der linken Hand an die Tafel zu schreiben und schrieb mit der rechten weiter, ohne daß sich das Schriftbild im geringsten geändert hätte; er konnte auch, wenn man ihm am Schreibtisch gegenüber saß, auf dem Kopf schreiben, und sogar Spiegelschrift schrieb er ebenso zügig wie Klarschrift.

Grötzsch, der an Armut von kaum einem Studenten überboten wurde - er hatte nur Holzschuhe und zerrissene Kleidung-, war schnell eine stadtbekannte Persönlichkeit: auf seinem Weg ins Institut trank "der Professor" in einer Bäckerei seinen dünnen Kaffee, aß sein trockenes Brötchen und las die Tageszeitung; dabei nickte er einmal ein und lehnte sich an den geheizten Ofen; das traurige Ergebnis war ein großes Loch in seinem besten Jackett, das ihm von seinen Eltern erst wenige Tage zuvor zum Austausch für ein undefinierbares Kleidungsstück aus Kriegstagen geschickt worden war; mein Angebot, einen mir bekannten Schneider um Hilfe zu bitten, lehnte er ab und half sich auf seine Weise: am nächsten Tag verdeckte ein aufgehefteter grüner Flicken den Schaden. Grötzsch wohnte am Galgenberg in einer winzigen Dachstube; der Weg dorthin war so steil, daß er bei Glätte auf Socken runterrutschte. Ich sah einmal, wie er mitten auf einer von US-Fahrzeugen stark befahrenen Kreuzung tief in Gedanken auf einem Bleistiftstummel kauend stehen blieb, bis ihn ein Schutzmann zum Bürgersteig führte; sicher war nicht nur die Mathematik, sondern auch die Unterernährung Ursache dieses Abschaltens.

Bei Grötzsch hörte ich „Konforme Abbildung“; als im Hörsaal plötzlich das Licht ausging, appellierte er an unser Abstraktionsvermögen und redete im Dunkeln weiter; nach einigen Minuten hörten wir das Geräusch von Kreide an der Wandtafel ... Herrlich waren seine eruptiven Sentenzen und dies war die schönste: Meine Damen und Herren! Das Hauptproblem der Mathematik lautet: „Gegeben ist der Beweis, gesucht ist der Satz!" Auch didaktischen Ansprüchen entsprach Grötzsch mit seiner bildhaften Diktion; ein kostbares Beispiel ist seine Erklärung zum "Satz von Bolzano": Denken Sie sich ein endliches Intervall und dadrin unendlich viele Punkte; da sagt einem doch schon die Anschauung: da muß es doch einen Punkt geben, wo ein ganz fürchterliches Gedränge stattfindet - und so ein Punkt, das ist ein „Häufungspunkt“! Als Grötzsch 1948 den Ruf nach Halle annahm, war die Fakultät, die wegen seiner zerlumpten Kleidung eine Verbesserung seiner Position als „unpassend“ abgelehnt hatte, fassungslos über diesen „Undank“. Wir Studenten aber hatten diesen liebenswerten Menschen ins Herz geschlossen! Krafft und Grötzsch haben in dieser Hungerzeit Übermenschliches geleistet!

Wirklich brauchbare Mathematik lernte ich in der Physik, wo mir bald der bekannte theoretische Physiker Erich Hückel, dessen 100. Geburtstag Stadt und Universität Marburg in 4 Monaten feiern werden, seine Hilfsassistentenstelle als "Hausmathematiker" anbot. Hückel war in seinen Vorlesungen so ungeschickt, daß er heute nur Kopfschütteln auslösen würde, aber sein Ringen um gedankliche Klarheit faszinierte uns so sehr, daß jeder ihm atemlos zuhörte; damals belastete der Schrei nach Didaktik eben noch nicht die geistige Nabelschnur ... Ich glaube, daß mir die herrliche Zusammenarbeit mit diesem ringenden und leidenden Menschen, aus der eine tiefe Freundschaft zwischen unseren beiden Familien erwuchs, den Blick für das Geschäft hat, was ich das ,soziale Problem der 
Mathematik" nenne: Nichtmathematikern Mathematik schmackhaft zu machen!

Ich komme zum Schluß: nach diesem ersten Marburger Semester, dem Sommersemester 1946, fuhr ich - jetzt endlich wieder mit der Bahn, aber 13 Stunden in uralten 3-achsigen fensterglaslosen überfüllten Waggons mit endloser Kontrolle in Eichenberg, wo die alliierte Militärpolizei offenbar flüchtige Nazis suchte, - wieder nach Hamburg, um mich mit der jungen Dame, die mich 5 Monate zuvor mit Blumen erfreut hatte, zu verloben.

Adresse des Autors:

Prof. Dr. Horst Tietz

Institut für Mathematik

Universität Hannover

Postfach 6009

30060 Hannover

\section{Leserbriefe}

\section{Ärgerliche 7 Jahre zum Ersten ...}

Stellungnahme zu dem Bericht von Herrn Vollrath in den Mitteilungen der DMV 1/1996 („Die ärgerlichen sieben Jahre“) und Presseecho und Stellungsnahmen dazu

Eine Anmerkung vorweg: ich würde mich heute nicht mehr als Mathematiker bezeichnen, obwohl ich einmal Mathematik studiert habe, sondern als Manager und damit Betroffener von Arbeitsergebnissen u.a. mathematisch ausgebildeter Kollegen und Mitarbeiter.

Die aufgeworfene Diskussion ist wichtig. Wenn fast alle Schüler Mathematik als dasjenige Fach bezeichnen, das ihnen in der Schule verhaßt war, so muß dies zu denken geben und auf einen tieferliegenden Grund verweisen. Diese Schulerfahrung hat zudem eine lange Tradition, d.h. sie ist Zeichen eines bereits langdauernden Mißstands. Deshalb fällt die Reaktion auf die Kritik von Herrn Heymann bei den Adressaten natürlich umso heftiger aus. Diese Reaktionen sind aber im allgemeinen ungerechtfertigt.

Wenn ich lese, daß die Mathematische Fakultät der Universität Bielefeld Stellungnahmen und Klarstellungen verschickt, und auch Herr Heymann sich genötigt sieht, dem wiederum Klarstellungen und Erläuterungen nachzureichen, so ist dieses Verhalten des Fachbereichs mir unverständlich und unangemessen. Herrn Heymanns Verhalten kann ich noch nachvollziehen, wenn ich mir klar mache, daß er in Bielefeld seine Habilitation zu einem guten Ende führen will und als unabhängig Beschäftigter auf das Wohlwollen einiger Kollegen angewiesen ist. Der ganze Vorgang spricht jedoch weder für die Souveränität und Standfestigkeit des Bielefelder Fachbereichs noch für die Fürsorgepflicht, die man als Vorgesetzte für seine Mitarbeiter hat. Die Kritik verkennt auch die Bedeutung einer Habilitationsschrift an einer deutschen Universität. Hier geht es nicht um ein Jahrhundertwerk, sondern um eine formale Qualifikation im (akademischen) Berufsleben. Diese Habilitation zeichnet sich vielleicht nicht durch besondere Tiefe mathematischer Gedanken aus, dafür aber durch die Wichtigkeit des Themas, eine Wichtigkeit, die auch in der (nichtmathematischen) Öffentlichkeit zur Kenntnis genommen wird (s. Pressemeldungen). Das unterscheidet diese Habilitationsschrift sicherlich von vielen anderen in der Mathematik. Die wichtigen Fragen sind also:

1. Welche Mathematik wird gelehrt (Inhalte) und

2. auf welche Weise (Didaktik)?

Damit wird es eine der wenigen mathematischen Habilitationsschriften sein, die überhaupt für die Öffentlichkeit Bedeutung haben. Ich habe mir den Kauf bereits vorgemerkt und erwarte mit Spannung ihr Erscheinen.

Daß viele Kollegen die Probleme, um die es Herrn Heymann geht, nicht verstanden haben, kann ich sowohl aus eigener Erfahrung als auch mit der Lektüre der Zuschriften zu diesem Thema in den DMVMitteilungen belegen.

Die letzte DMV-Tagung, die ich besucht habe, war - nach langer Pause - 1991 in Bielefeld. Es hatte sich nichts verändert. Es gab wieder eine kleine Sektion zur Industriemathematik. Der Vortrag eines Kollegen war dort sehr gut besucht; er sprach über ausgewählte Projekte und die mathematischen Probleme, die darin zu lösen waren. Es war ein Bericht aus seiner Praxis, jetzt aber nicht als Hochschullehrer, der er hauptberuflich war, sondern als nebenberuflicher Unternehmensberater. Viele Aussagen dieses Kollegen wurden im Auditorium von Lächeln oder auch Gelächter begleitet, für mich eine frappierende Erfahrung des Unverständnisses, das von den Vertretern der (reinen) Mathematik diesem Kollegen entgegengebracht wurde. Ihnen war die verwendete Mathematik und ihre Präsentation auf einer Tagung zu trivial. Inhaltlich ging es im Wesentlichen um Optimierungsprobleme mit vielen Randbedingungen und Parametern, und es mußten zum Schluß bei einer Zielfunktion absolute Minima gesucht werden. Ich kann mich noch erinnern an ein Beispiel aus der Kaffeeröstung, wo beim Einsatz unterschiedlicher Rohstoffe (Kaffeesorten aus mehreren Ländern mit ver- 